Original Research Paper

\title{
Circulation of Multidrug-Resistant (MDR) and Extensively Drug-Resistant (XDR) Mycobacterium tuberculosis Strains in Mexico
}

\author{
${ }^{1}$ Carlos A. Vazquez-Chacon, ${ }^{1,2}$ Armando Martinez-Guarneros, \\ ${ }^{1}$ Susana Balandrano Campos, ${ }^{2}$ Julieta Luna Herrera, ${ }^{1}$ Claudia Bäcker, \\ ${ }^{3}$ Livia Maria Gonçalves Rossi, ${ }^{1}$ Karina Ruiz-Tovar, ${ }^{4}$ Salvador Fonseca-Coronado, \\ ${ }^{1}$ Juan C. Carpio-Pedroza, ${ }^{1}$ Gilberto Vaughan and ${ }^{1}$ Alejandro Escobar-Gutierrez \\ ${ }^{1}$ Instituto de Diagnóstico y Referencia Epidemiológicos, Secretaría de Salud, México City, México \\ ${ }^{2}$ Escuela Nacional de Ciencias Biológicas, Instituto Politécnico Nacional, México City, México \\ ${ }^{3}$ Department of Biology, Institute of Bioscience, \\ Language and Exact Science, São Paulo State University, São José do Rio Preto, SP, Brazil \\ ${ }^{4}$ Unidad de Investigación Multidisciplinaria, \\ Facultad de Estudios Superiores Cuautitlán, Universidad Nacional Autónoma de México, Estado de México, México
}

Article history

Received: 2014-10-24

Revised: 2014-10-28

Accepted: 2014-12-26

Corresponding Author: Carlos A. Vazquez-Chacon, Instituto de Diagnóstico y Referencia Epidemiológicos, Secretaría de Salud,

México City, México

Email: cavchacon@gmail.com

\section{Introduction}

Tuberculosis (TB) is caused by the infection of Mycobacterium tuberculosis. This is an airborne infectious disease and a major public health problem worldwide (Ahmad, 2014) World Health Organization (WHO) estimated 8.6 million people infected with M. tuberculosis and 1.3 million deaths in 2012 (WHO, 2013). In 2013, WHO declared Multidrug-Resistant TB (MDR-TB) as a public health crisis and estimated $\sim 450,000$ people affected by MDR-TB with $\sim 170,000$ MDR-TB deaths in 2012 (WHO, 2014). The emergence of MDR and Extensively Drug-Resistant (XDR) TB brings several concerns and jeopardize the efficacy of strategies aimed to control disease spread. MDR is a form of TB which is characterized by resistance at least to isoniazid $(\mathrm{H})$ and Rifampicin (R). Besides resistance to $\mathrm{H}$ and $\mathrm{R}$, XDR strains are also resistant to any of the fluoroquinolones (such as Ofloxacin [Ofx] or Ciprofloxacin [Cip]) and to at least one of three injectable second-line drugs (amikacin [Amk], Kanamycin [Km] or Capreomycin [Cm]) (WHO, 2011).

The development of drug resistance is commonly due to misuse and mismanage of anti-TB drugs (Shin et al., 2010). Alternatively, XDR-TB can also be transmitted from individuals carrying drug resistant strains. XDR-TB therapy is challenging, expensive and the likelihood of success is much lower than in patients with ordinary TB or even MDR-TB (Haydel, 2010). Treatment success rate for MDR-TB is about $60 \%$; however, the success of therapy is reduced to half for XDR-TB (Kim et al., 2008). Moreover, MDR and XDR-TB require longer treatment and are usually accompanied by more severe side effects (Caminero et al., 2010).

Different studies conducted in Latin-American countries have shown the increase of MDR-TB (Pelly et al., 2004). The rising of drug resistance in Latin-America and other parts of the world (Migliori et al., 2010), highlights the need of national efforts and international cooperation to implement strategies for TB control. Interestingly, rates of latent TB along the Mexico-USA border have been found to be $21.8 \%$ in Latino versus $5.6 \%$ for non-Latino individuals (Sipan et al., 2003). Likewise, young Latinos living near the border are at higher risk for undiagnosed infection and inadequate treatment. Incompleteness of treatment among Latino individuals has been associated with idiosyncrasy and cultural barriers that
Science

Publications 
play role in adherence and ultimately in treatment success (Kandula et al., 2004). Interestingly, Hispanic patients born outside the US or Mexico were more likely to have MDR-TB than were those born within these countries (Bojorquez et al., 2013).

In this study, we assessed the circulation of XDR-TB isolates in Mexico. The presence of Pre-XDR strains in different geographical regions of the country highlights the importance of surveillance for the appropriate monitoring of drug resistant strains. within parentheses.

\section{Materials and Methods}

Here, we analyzed 137 epidemiologically non-related M. tuberculosis isolates associated with drug resistance collected via the State Laboratory Network in Mexico during 2006 and 2008. Colony morphology and acid-fast bacilli identification on smears by Ziehl-Neelsen technique and culture in Löwenstein-Jensen solid slants were performed. Biochemical analyses for differentiation included nitrate reduction, niacin accumulation test and catalase test both 37 and $68^{\circ} \mathrm{C}$. All isolates were subjected to routine culture-based drug susceptibility testing to determine the drug resistance patterns. Susceptibility profiles to first line drugs were determined by the fluorometric method MGIT 960 (Becton Dickinson, Sparks, MD). Susceptibility testing to second line drugs (Amk, Km, Cm, Ofx and Cip), was evaluated by the Proportions Method. Mycobacterial strains were classified into three groups according to their resistance profile. MDR, Pre-XDR (MDR strains also resistant to either a fluoroquinolone or a second-line injectable agent but not both) and XDR-TB. Additionally, resistance to Streptomycin (S), pyrazinamide (Z), Ethambutol (E) and Ethionamide (Eto) was assessed.

\section{Results}

The clinical samples were originally obtained from 26 different states in Mexico (Fig. 1 and Table 1). The patterns of drug-resistance showed that $80(58 \%)$ isolates were exclusively MDR, while 45 (33\%) and 12 (9\%) were classified as Pre-XDR and XDR, respectively. Overall, resistance to fluoroquinolones $(25.5,21.2 \%$ for Ofx and Cip, respectively) was more frequent than resistance to Amk (11\%), Km (19\%) and Cm (8.7\%). This pattern was also observed among Pre-XDR strains where resistance to fluoroquinolones was also more common (Table 1).

In general, resistance among Pre-XDR strains was evenly distributed between strain resistant to fluoroquinolones (22) and injectable drugs (23). Twenty Pre-XDR isolates exhibited resistance to only one fluoroquinolone (3) or one injectable drug (17). Twenty strains were resistant to both fluoroquinolones and only one isolate was resistant to Amk and $\mathrm{Km}$. Additionally, four isolates displayed resistance to all injectable drugs. All twelve XDR strains were resistant to Ofx and only three were susceptible to
Cip. Most XDR strains were also resistant to $\mathrm{Km}$ and only half to Amk. Resistance to $\mathrm{Cm}$ was observed among 5 isolates.

Overall resistance to other first-line drugs ranged between $35-39 \%$ (37.2, 38.7 and $35.8 \%$ for $S, Z$ and $E$, respectively). Resistance to Eto was slightly higher (39.4\%). Resistance to first-line drugs was slightly higher among Pre-XDR isolates than MDR strains. The small number of XDR samples did not allow establishing resistance patterns (Table 2). However, resistance to Eto was observed in almost all XDR strains, while $\sim 30 \%$ of the MDR and Pre-XDR isolates showed resistance to Eto (Table 2).

\section{Discussion}

In this study, we have shown the characteristic drug resistance patterns of $M$. tuberculosis strains circulating in Mexico. The results showed the increasing problem with Pre-XDR and XDR-TB. In Mexico, different studies have reported the presence of MDR strains (ZazuetaBeltran et al., 2011, Bojorquez-Chapela et al., 2013). Circulation of XDR strains in the country has also been suggested (Banerjee et al., 2008). Here, a significant number of Pre-XDR strains were observed. The circulation of such resistant strains is a major concern considering that acquisition of resistance to a second-line anti-TB agent is highly possible in an environment where inappropriate treatment management is frequently observed (Laniado-Laborín and Cabrales-Vargas, 2000).

Table 1. Strain distribution per state

\begin{tabular}{ll}
\hline State & N $(\%)$ \\
\hline AGU & $3(2.2)$ \\
BCN & $2(1.5)$ \\
BCS & $5(3.6)$ \\
CAM & $3(2.2)$ \\
CHH & $7(5.1)$ \\
CHP & $16(11.7)$ \\
COA & $2(1.5)$ \\
COL & $3(2.2)$ \\
GRO & $2(1.5)$ \\
GTO & $7(5.1)$ \\
HGO & $11(8)$ \\
JAL & $8(5.8)$ \\
MIC & $2(1.5)$ \\
MOR & $7(5.1)$ \\
NAY & $1(0.7)$ \\
NLE & $7(5.1)$ \\
OAX & $6(4.4)$ \\
PUE & $6(4.4)$ \\
ROO & $3(2.2)$ \\
SIN & $5(3.6)$ \\
SON & $4(2.9)$ \\
TAB & $2(1.5)$ \\
TAM & $4(2.9)$ \\
VER & $17(12.4)$ \\
YUC & $3(2.2)$ \\
ZAC & $1(0.7)$ \\
\hline
\end{tabular}




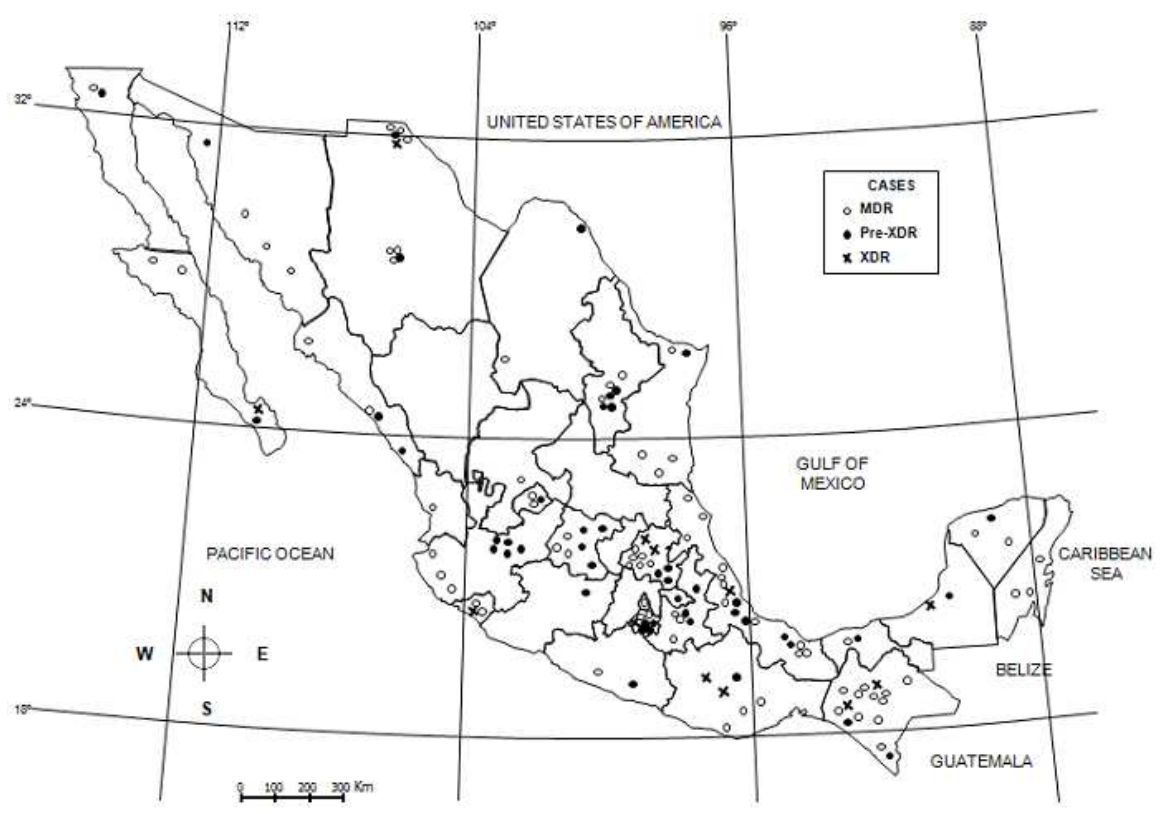

Fig. 1. States from which resistant Mycobacterium tuberculosis isolates were recovered are indicated on the map. MDR, Pre-XDR and XDR-TB isolates are depicted on the map

Table 2. TB strains resistance patterns

$\frac{\text { Table 2. TB strains }}{\mathrm{N} \%}$

\begin{tabular}{|c|c|c|c|c|c|c|c|c|c|c|}
\hline & \multicolumn{4}{|c|}{ First-line } & \multicolumn{6}{|c|}{ Second-line } \\
\hline & $\mathrm{N}$ & $\mathrm{S}$ & $\mathrm{Z}$ & $\mathrm{E}$ & Amk & $\mathrm{Km}$ & $\mathrm{Cm}$ & Ofx & Cip & Eto \\
\hline$\overline{\mathrm{MDR}}$ & 80 & $29(36.2)$ & $28(35)$ & $25(31.2)$ & & & & & & $26(32.5)$ \\
\hline Pre-XDR & 45 & $20(44.4)$ & $22(48.8)$ & $21(46.6)$ & $9(20)$ & $15(33.3)$ & $7(15.5)$ & $23(51.1)$ & $20(44.4)$ & $17(37.7)$ \\
\hline XDR & 12 & $2(16.6)$ & $3(25)$ & $3(25)$ & $6(50)$ & $11(91.6)$ & $5(41.6)$ & $12(100)$ & $9(75)$ & $11(91.6)$ \\
\hline
\end{tabular}

Pre-XDR-TB strains have been reported to circulate in the USA, China, South Africa and Korea (Banerjee et al., 2008; Kim et al., 2010; Qi et al., 2012; Van Der Plas et al., 2011). The presence of these Pre-XDR-TB strains in different parts of the world highlights the importance of timely diagnosis aimed to prevent the development of XDR-TB.

While in most bacteria drug resistance is usually acquired by horizontal transfer of mobile genetic elements (plasmids, transposons or integrons), in the case of M. tuberculosis acquisition of resistance is mediated primarily by spontaneous mutations in chromosomal genes, which are selected under sub-optimal therapy regimes (Kochi et al., 1993). Thus, drug resistance has been associated with several mycobacterial genes (Kozhamkulov et al., 2011). It is noteworthy that classical mutations associated with resistance to a given drug could initiate resistance towards other drugs (Safi et al., 2008), as in the case of resistance to $\mathrm{H}$ which is also related to resistance to Eto (Banerjee et al., 1994). Therefore, it is not surprising the high degree of resistance to Eto observed among all these MDR-TB strains. Likewise, the presence of Pre-XDR strains is a latent risk for development of XDR strains.
Cross-resistance to fluoroquinolones is commonly assumed; however, the presence of mutations conferring resistance to particular fluoroquinolones has also been reported (Von Groll et al., 2009). Here, four isolates with resistance to Ofx but sensitive to Cip were identified. However, the molecular bases responsible for this resistant phenotype were not assessed. Crossresistance between second-line injectable drugs has also been shown (Alangaden et al., 1998). Conversely, other studies have suggested that $\mathrm{Km}$ and Amk crossresistance is not absolute, showing variable patterns and levels of resistance (Kruuner et al., 2003). Our results showed a high correlation between resistance to $\mathrm{Km}$ and both Amk and $\mathrm{Cm}$. Importantly, universal cross-resistance to second-line injectable agents was not observed.

The main limitation of this study is the likelihood of underestimating the actual number of XDR-TB since complete drug susceptibility testing (other fluoroquinolones such as moxifloxacin) was not performed. Therefore, we cannot rule out resistance to other fluoroquinolones. Also, underreporting is a major concern because most likely not all TB cases are identified by the current surveillance system. Finally, the characterization of the mutations 
responsible for drug resistance among these specimens further limits our understanding on the molecular basis controlling TB drug resistance.

\section{Conclusion}

In conclusion, it is of the utmost importance to evaluate the drug resistance profile, as a compulsive component in the therapeutic monitoring of TB patients. Identification of resistance patterns against the first and second-line of anti-TB drugs in Mexico will contribute to develop better strategies for disease control. Surveillance and drug susceptibility testing should be improved in Mexico to monitor the circulation of MDR, Pre-XDR and XDR-TB strains.

\section{Acknowledgement}

This study was supported by project Salud 2010-C01139760, CONACYT.

\section{Author's Contributions}

Carlos A. Vazquez-Chacon and Armando MartinezGuarneros contributed equally in this study.

\section{Ethics}

The authors of this manuscript declare no conflict of interest.

\section{References}

Alangaden, G.J., B.N. Kreiswirth, A. Aouad, M. Khetarpal and F.R. Igno et al., 1998. Mechanism of resistance to amikacin and kanamycin in Mycobacterium tuberculosis. Antimicrob Agents Chemother, 42: 1295-1297.

Ahmad, S.M.E., 2014. Current status and future trends in the diagnosis and treatment of drug-susceptible and multidrug-resistant tuberculosis. J. Infect. Public Health, 7: 75-91. DOI: 10.1016/j.jiph.2013.09.001

Banerjee, A., E. Dubnau, A. Quemard, V. Balasubramanian and K.S. Um et al., 1994. inhA, a gene encoding a target for isoniazid and ethionamide in Mycobacterium tuberculosis. Science, 263: 227230. DOI: $10.1126 /$ science. 8284673

Banerjee, R., J. Allen, J. Westenhouse, P. Oh and W. Elms et al., 2008. Extensively drug-resistant tuberculosis in California, 1993-2006. Clin. Infect. Dis., 47: 450-457. DOI: 10.1086/590009

Bojorquez, I., R.F. Barnes, J. Flood, H. López-Gatell and T.C. Rodwell, 2013. Multidrug-resistant tuberculosis among patients in Baja California, Mexico and Hispanic patients in California. Am. J. Public Health, 103: 1301-5. DOI: 10.2105/AJPH.2012.301039
Bojorquez-Chapela, I., C.E. Bäcker, I. Orejel, A. López and H. López-Gatell, 2013. Drug resistance in Mexico: Results from the national survey on drug-resistant tuberculosis. Int. J. Tuberculosis Lung Dis., 17: 514519. DOI: $10.5588 /$ ijtld. 12.0167

Caminero, J.A., G. Sotgiu, A. Zumla and G.B. Migliori, 2010. Best drug treatment for multidrug-resistant and extensively drug-resistant tuberculosis. Lancet Infect. Dis., 10: 621-629.

DOI: 10.1016/S1473-3099(10)70139-0.A

Haydel, S.E., 2010. Extensively drug-resistant tuberculosis: A sign of the times and an impetus for antimicrobial discovery. Pharmaceuticals, 3: 22682290. DOI: 10.3390/ph3072268

Kochi, A., B. Vareldzis and K. Styblo, 1993. Multidrugresistant tuberculosis and its control. Res. Microbiol., 144: 104-110. DOI: 10.1016/0923-2508(93)90023-U

Kruuner, A., P. Jureen, K. Levina, S. Ghebremichael and S. Hoffner, 2003. Discordant resistance to kanamycin and amikacin in drug-resistant Mycobacterium tuberculosis. Antimicrobial Agents Chem., 47: 2971-2973.

DOI: 10.1128/AAC.47.9.2971-2973.2003

Kandula, N.R., M.S. Dworkin, M.R. Carroll and D.S. Lauderdale, 2004. Tuberculosis prevention in Mexican immigrants: Limitations of short-course therapy. Am. J. Prev. Med., 26: 163-166. DOI: $10.1016 / \mathrm{j}$. amepre.2003.10.011

Kim, D.H., H.J. Kim, S.K. Park, S.J. Kong and Y.S. Kim et al., 2008. Treatment outcomes and longterm survival in patients with extensively drugresistant tuberculosis. Am. J. Respir. Crit. Care Med., 178: 1075-1082. DOI: 10.1164/rccm.200801-132OC

Kim, D.H., H.J. Kim, S.K. Park, S.J. Kong and Y.S. Kim et al., 2010. Treatment outcomes and survival based on drug resistance patterns in multidrug-resistant tuberculosis. Am. J. Respir. Crit. Care Med., 182: 113-119.

DOI: $10.1164 / \mathrm{rccm} .200911-1656 \mathrm{OC}$

Kozhamkulov, U., A. Akhmetova, S. Rakhimova, E. Belova and A. Alenova et al., 2011. Molecular characterization of rifampicin- and isoniazidresistant Mycobacterium tuberculosis strains isolated in Kazakhstan. Jpn. J. Infect. Dis., 64: 253255. PMID: 21617314

Laniado-Laborín, R. and N. Cabrales-Vargas, 2000. Tratamiento acortado estrictamente supervisado: Estrategia necesaria, pero no suficiente para controlar la tuberculosis en Baja California, México. Elevadas tasas de multidrogorresistencia en el estado/directly observed shortened treatment a necessary but insufficient strategy to control tuberculosis in Baja California, Mexico. High rate of multidrugresistance in the state. Rev. Inst. Nac. Enfermedades Respir., 13: 23-27. PMID: 280330 
Migliori, G.B., R. Centis, C. Lange, M.D. Richardson and G. Sotgiu, 2010. Emerging epidemic of drugresistant tuberculosis in Europe, Russia, China, South America and Asia: Current status and global perspectives. Current Opinion Pulmonary Med.., 16: 171-179. DOI: 10.1097/MCP.0b013e328337573e

Pelly, T., D.A. Moore, R. Gilman and C. Evans, 2004. Recent tuberculosis advances in Latin America. Current Opinion Infect. Dis., 17: 397-403.

Qi, Y.C., M.J. Ma, D.J. Li, M.J. Chen and Q.B. Lu et al., 2012. Multidrug-resistant and extensively drugresistant tuberculosis in multi-ethnic region, Xinjiang Uygur Autonomous Region, China. PLoS One. DOI: 10.1371/journal.pone.0032103

Sipan, C., E. Blumberg, M. Hovell, N. Kelley and K. Moser et al., 2003. Screening Latino adolescents for Latent Tuberculosis Infection (LTBI). Public Health Rep, 118: 425-433.

Safi, H., B. Sayers, M.H. Hazbon and D. Alland, 2008. Transfer of embB codon 306 mutations into clinical Mycobacterium tuberculosis strains alters susceptibility to ethambutol, isoniazid and rifampin. Antimicrobial Agents Chem., 52: 2027-2034. DOI: 10.1128/AAC.01486-07

Shin, S.S., S. Keshavjee, I.Y. Gelmanova, S. Atwood and M.F. Franke et al., 2010. Development of extensively drug-resistant tuberculosis during multidrug-resistant tuberculosis treatment. Am. J. Respir. Crit. Care Med., 182: 426-432. DOI: $10.1164 / \mathrm{rccm} .200911-1768 \mathrm{OC}$
Van Der Plas, H. and M. Mendelson, 2011. High prevalence of comorbidity and need for up-referral among inpatients at a district-level hospital with specialist tuberculosis services in South Africa: The need for specialist support. S. Afr. Med. J., 101: 529-532. PMID: 21920125

Von Groll, A., A. Martin, P. Jureen, S. Hoffner and P. Vandamme et al., 2009. Fluoroquinolone resistance in Mycobacterium tuberculosis and mutations in gyr $A$ and gyrB. Antimicrobial Agents Chem., 53: 4498-4500. DOI: 10.1128/AAC.00287-09

WHO, 2011. Towards universal access to diagnosis and treatment of multidrug-resistant and extensively drug-resistant tuberculosis by 2015 . World Health Organization.

WHO, 2013. Executive sumary, global tuberculosis report. $\mathrm{WHO} / \mathrm{HTM} / \mathrm{TB} / 2013.15$

WHO, 2014. Reaching people with MDR-TB, Progress in diagnosis, a key step in overcoming the MDR-TB crisis.

Zazueta-Beltran, J., N. Leon-Sicairos, S. Muro-Amador, A. Flores-Gaxiola and J. Velazquez-Roman et al., 2011. Increasing drug resistance of Mycobacterium tuberculosis in Sinaloa, Mexico, 1997-2005. Int. J. Infect. Dis., 15: e272-276.

DOI: $10.1016 /$ j.ijid.2011.01.001 